\title{
Small and round seed 5 gene encodes alpha- tubulin regulating seed cell elongation in rice
}

\author{
Shuhei Segami ${ }^{1}$, Izumi Kono ${ }^{2,3}$, Tsuyu Ando ${ }^{2,4}$, Masahiro Yano ${ }^{5}$, Hidemi Kitano ${ }^{6}$, Kotaro Miura ${ }^{{ }^{*}}$ and \\ Yukimoto Iwasaki ${ }^{*}$
}

\begin{abstract}
Seed size is an important trait in determinant of rice seed quality and yield. In this study, we report a novel semidominant mutant Small and round seed 5 (Srs5) that encodes alpha-tubulin protein. Lemma cell length was reduced in Srs5 compared with that of the wild-type. Mutants defective in the G-protein alpha subunit (d1-1) and brassinosteroid receptor, BRI1 (d61-2) also exhibited short seed phenotypes, the former due to impaired cell numbers and the latter due to impaired cell length. Seeds of the double mutant of Srs 5 and $d 61-2$ were smaller than those of Srs5 or d61-2. Furthermore, SRS5 and BR/1 genes were highly expressed in Srs5 and d61-2 mutants. These data indicate that SRS5 independently regulates cell elongation of the brassinosteroid signal transduction pathway
\end{abstract}

\section{Background}

Seed size and weight are important traits for rice yield (Song and Ashikari 2008, Takeda and Matsuoka 2008). Several quantitative trait loci (QTLs) affecting seed size have been identified, namely GW2 encoding a RINGtype protein that functions as an E3 ubiquitin ligase (Song et al. 2007), qSW5 encoding a novel protein with no known domains (Shoumura et al. 2008), and GS3 encoding a membrane protein with various conserved domains (Fan et al. 2006, Takano-Kai et al. 2009). Loss of GW2 and qSW5 function leads to a wider seed phenotype, and loss of GS3 function leads to a longer seed phenotype, both resulting in increased yield.

Causal genes of the small (or short) seed mutants have also been identified, namely $d 1$ (also named RGA1) encoding the heterotrimeric $G$ protein alpha subunit (Ashikari et al. 1999, Fujisawa et al. 1999), d11 encoding a cytochrome P450 involved in brassinosteroid (BR) biosynthesis (Tanabe et al. 2005), $d 2$ and brd2 encoding another type of cytochrome P450 involved in BR synthesis (Hong et al. 2003, Hong et al. 2005), d61 (also named OsBRI1) encoding the BR receptor (Yamamuro et al. 2000), srs1 encoding a novel protein that has no known functional domains (Abe et al. 2010), and finally,

\footnotetext{
* Correspondence: miura-k@fpu.ac.jp; iwasaki@fpu.ac.jp

${ }^{1}$ Faculty of Biotechnology, Fukui Prefectural University, 4-1-1 Kenjojima,

Matsuoka, Eiheiji-Town, Fukui 910-1195, Japan

Full list of author information is available at the end of the article
}

srs3 encoding a kinesin 13 protein (Kitagawa et al. 2010). During seed formation in rice, it was demonstrated that $D 1$ regulates cell number (Izawa et al. 2010), and SRS1 and SRS3 regulate cell length (Abe et al. 2010, Kitagawa et al. 2010). From these observations, SRS1 and SRS3 seem to affect seed size through signaling pathways other than G-protein signal transduction.

Although several genes regulating seed size have been identified, their molecular network underlying seed formation remains unclear. Here we report molecular cloning of a novel small and round seed mutant in Srs 5 (Small and round seed 5). The results clearly demonstrated that $\operatorname{Srs} 5$ encodes alpha-tubulin and regulates cell elongation in rice seed.

\section{Results}

\section{Characterization of the Srs5 mutant}

A mutant line, Kyudai No. 37, was identified by screening of small or short seed mutants from the rice collections of Togo Field, Nagoya University, and renamed Small and round seed 5, (Srs5). Srs5 shows shorter and rounder seeds, a shorter panicle and semi-dwarf plant phenotype, compared to WT (Figure 1A-C). Additionally, $\mathrm{F}_{1}$ plants derived from a cross between WT and Srs 5 plants show intermediate seed length of parents seeds (Figure 1A and 1D). From these results, we presumed that the Srs 5 mutation acts as semi-dominant 

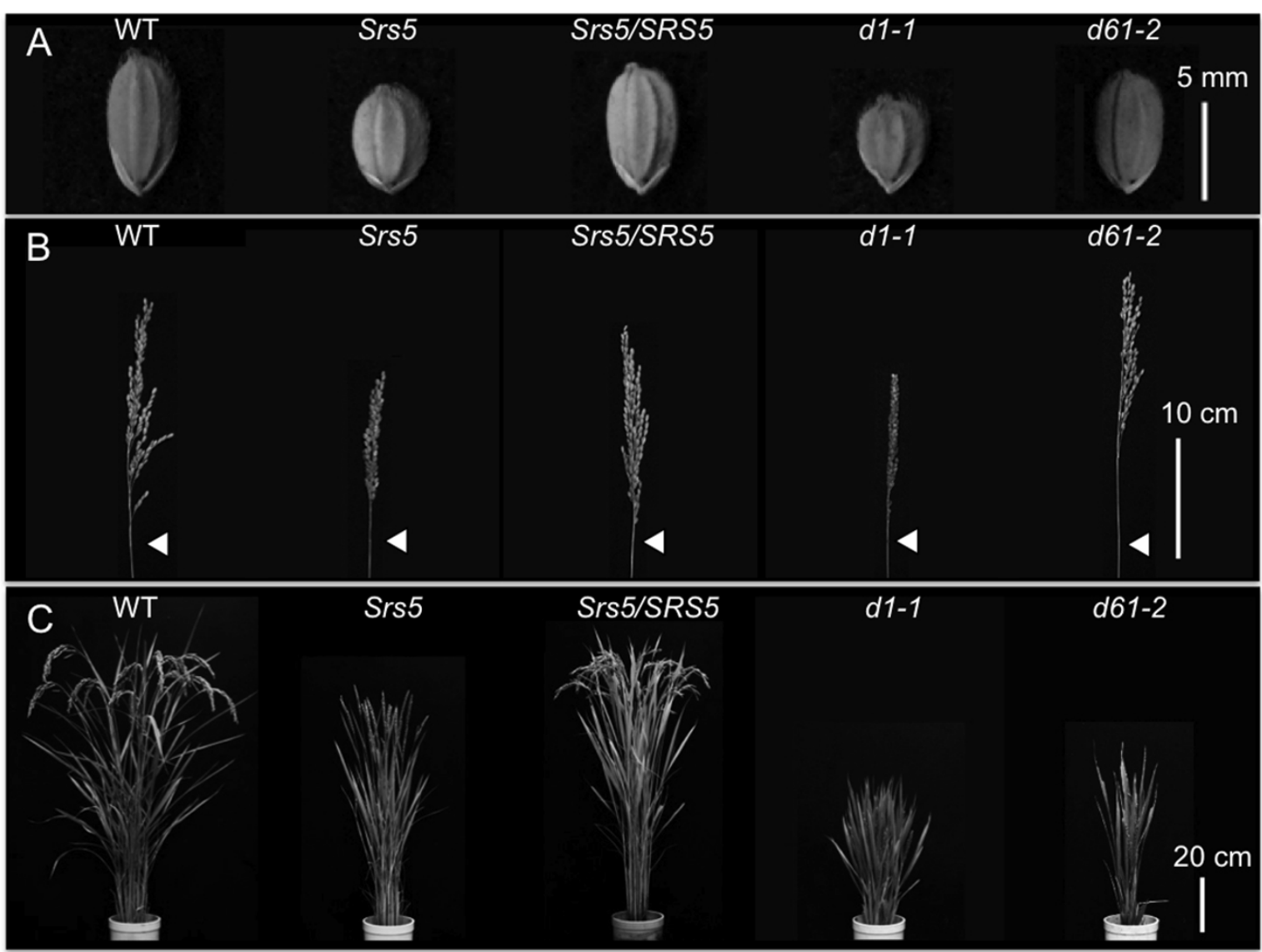

D

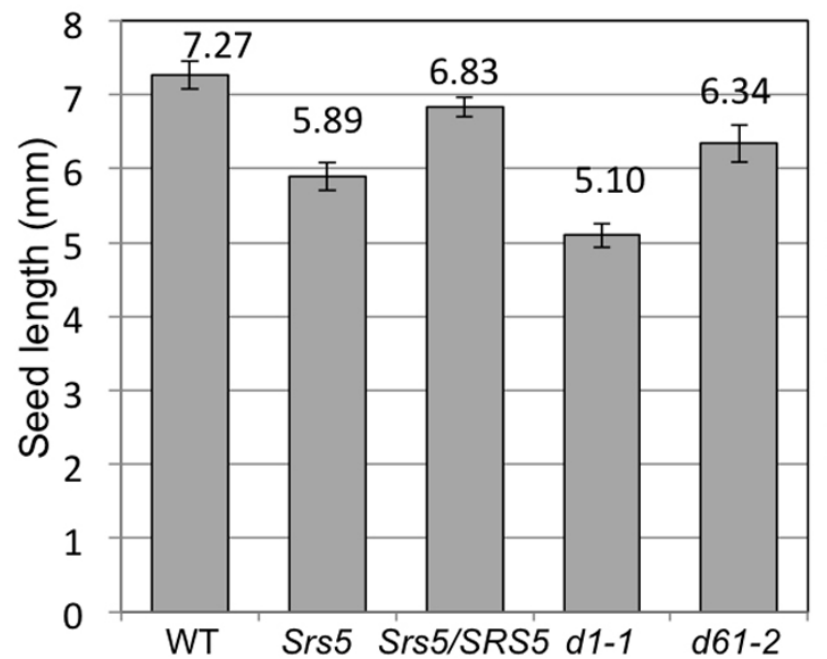

E

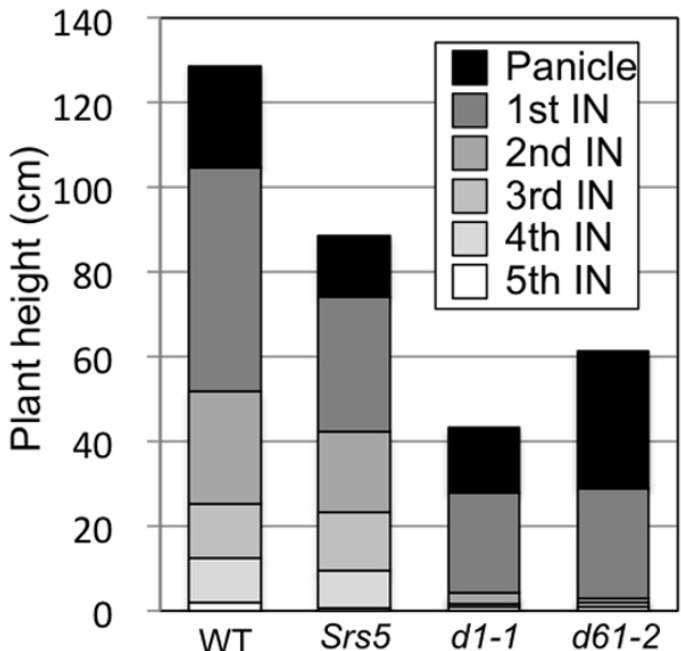

Figure 1 Srs 5 mutant phenotypes. (A) Seed morphology of T65, Srs5, Srs5/SRS5, d1-1, and d61-2. Bar = $5 \mathrm{~mm}$. (B) Panicle morphology of T65, Srs5, Srs5/SRS5, d1-1, and d61-2. Arrowheads indicate panicle neck nodes. Bar $=10 \mathrm{~cm}$. (C) Gross morphology of T65, Srs5, Srs5/SRS5, d1-1, and d61-2. Bar $=20 \mathrm{~cm}$. (D) Seed length of T65, Srs5, Srs5/SRS5, d1-1, and d61-2. Numbers on graphs indicate average seed length \pm S.D. (E) Internode length relative to the total length of the culm. Schematic representation of the internode elongation pattern of T65, Srs5, d1 1 1, and d61-2. IN: internode. 
gene. These phenotypes, short seed, short panicle, and dwarfism, are also exhibited by $d 1-1$ and $d 61-2$ mutants (Figure 1A-D). Comparison of internode elongation patterns among Srs5, $d 1-1$, and $d 61-2$ revealed that the internode elongation pattern of Srs 5 differs from that of $d 1-1$ and $d 61-2$ (Figure 1E). Although $d 1-1$ and $d 61-2$ exhibit extremely stunted second, third, and fourth internodes, Srs 5 shows equally shortened internodes (Figure 1E).

To characterize short seed phenotype of Srs 5 in detail, we compared the length of the inner epidermal cells of lemmas of Srs5, $d 1-1$, and d61-2 using scanning electron microscopy (SEM). The cells of Srs 5 were shorter than those of the WT (Figure 2A, B, and 2F), and similar to those of $d 61-2$ (Figure 2D and 2F), but not those of d1-1 (Figure 2C and 2F). Additionally, we estimated cell numbers by dividing lemma length (Figure 2E) and by cell length (Figure 2F). Although $d 1-1$ had a reduced number of inner epidermal cell of the lemma, the cell numbers of Srs5 and d61-2 were not significantly different from that of the WT (Figure 2G). From these observations, we concluded that the cause of the short seed phenotype of Srs5 is reduced cell length, as in $d 61-2$.

\section{SRS5 gene encodes alpha-tubulin}

To map the Srs 5 locus on rice chromosomes, we performed linkage analysis using $\mathrm{F}_{2}$ plants derived from a cross between the Srs 5 mutant (Oryza sativa. ssp.
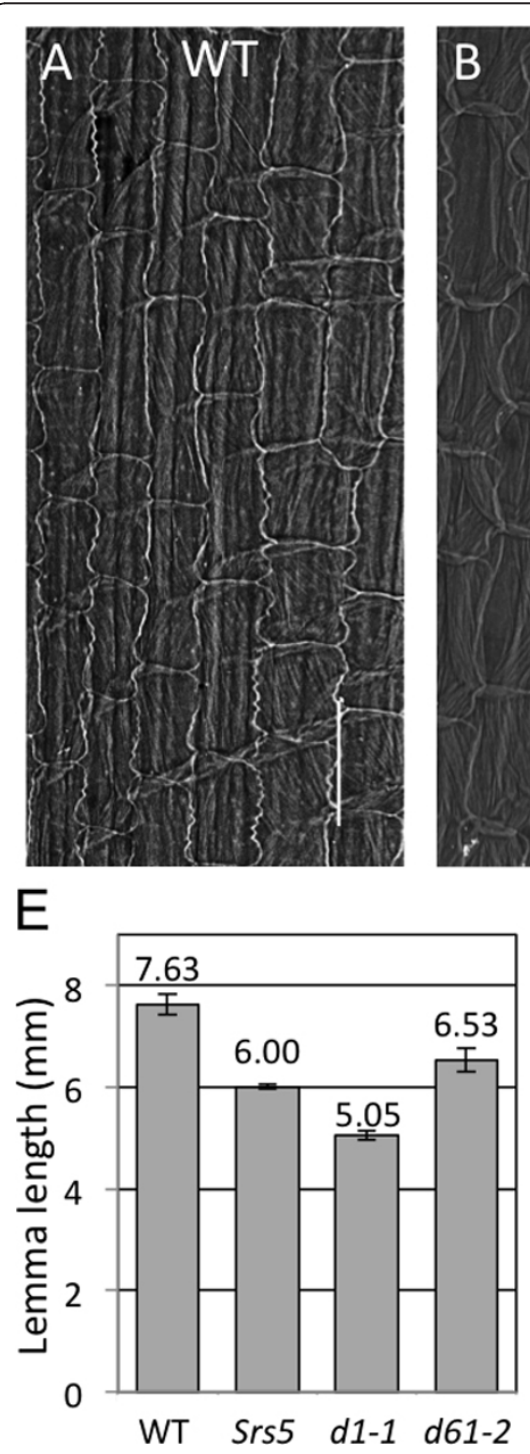

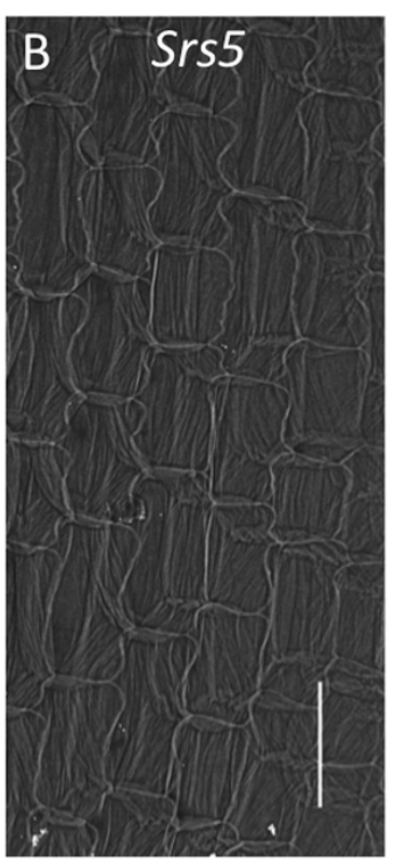

F

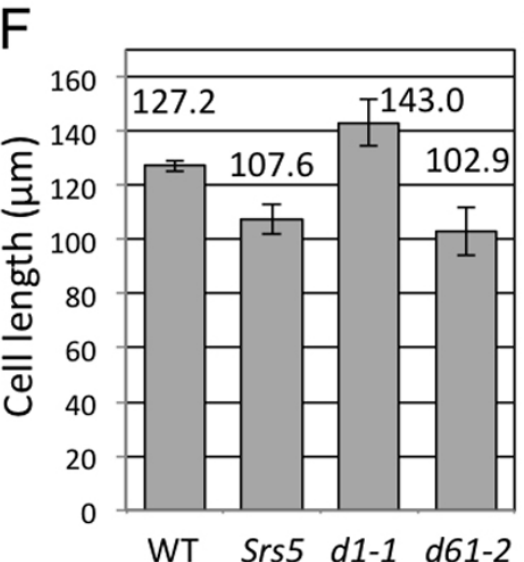

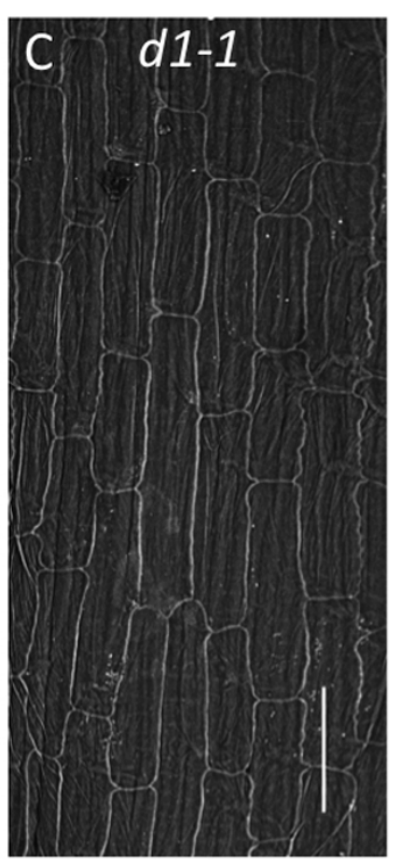
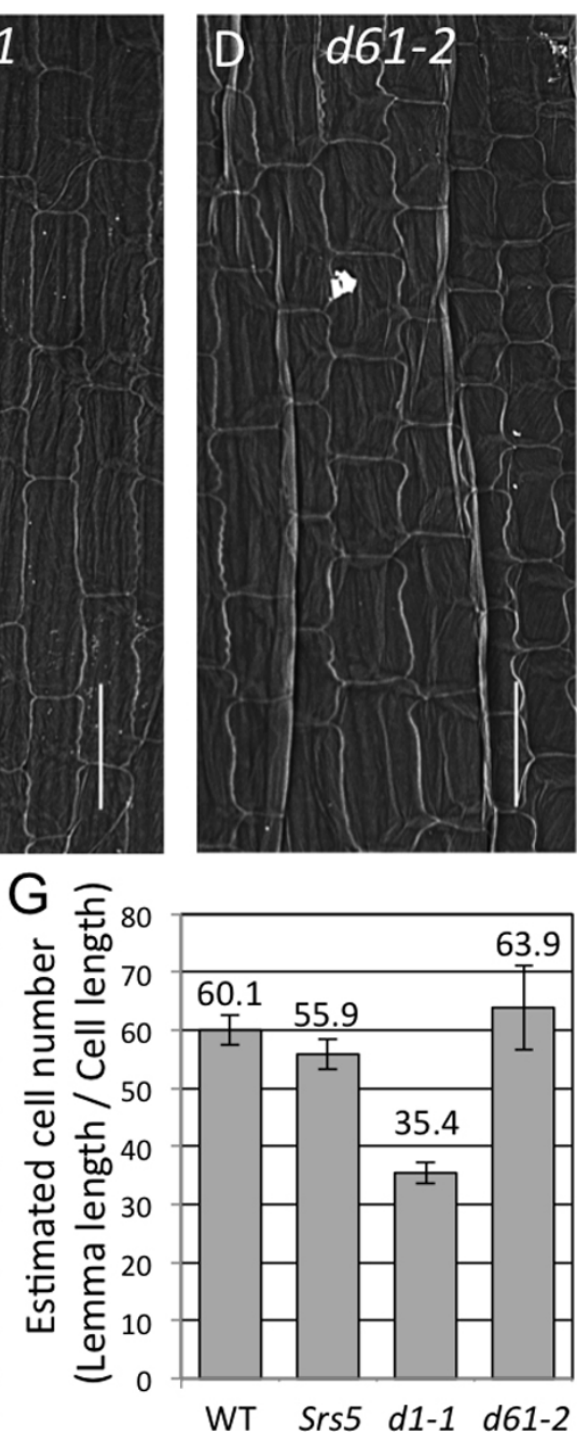

Figure 2 Comparison of inner epidermal cell length of T65, Srs5, $\boldsymbol{d} \mathbf{1} \mathbf{- 1}$, and $\mathbf{d} \mathbf{6} \mathbf{1 - 2}$. Inner epidermal cells of the lemma of WT (A), Srs5 (B), d1-1 (C), and d61-2 (D) observed by SEM. Bars = $100 \mu \mathrm{m}$. (E) Lemma length of the WT, Srs5, d1-1, and d61-2. (F) Inner epidermal cell length of WT, Srs5, d1-1, and d61-2. (G) Estimated cell numbers of WT, Srs5, d1-1, and d61-2. Numbers in (E-G) indicate averages \pm S.D. 
japonica) and Kasalath (Oryza sativa. ssp. indica). Since $\mathrm{F}_{2}$ seeds show a continuous variation in seed length, short seed phenotypic expression of seed size seems to be affected by difference in genetic background between the japonica and indica subspecies, in addition to Srs5 locus (Figure 3A). From $2000 \mathrm{~F}_{2}$ plants, we obtained 13 $\mathrm{F}_{2}$ plants that produced evident short seeds in $\mathrm{F}_{3}$ progeny, indicating homozygous of Srs 5 mutant gene. Linkage analysis revealed that Srs5 was located in the 1.8 Mb between chr11-7240 and chr11-9030 on chromosome 11 (Figure 3B). To further fine mapping of the gene, it was difficult to perform linkage analysis using this population, because of small number of plants with homozygous of mutant allele (small seed). For further analysis, we produced an $\mathrm{F}_{2}$ population derived from a cross between Srs5 and a Chromosome Segment Substitution Line (CSSL), SL233 that possessed the Kasalath chromosome segment of the short arm of chromosome 11 in a Koshihikari chromosome background. The phenotypes of $\mathrm{F}_{3}$ seeds obtained from these $\mathrm{F}_{2}$ plants were clearly distinguished mutant and wild types. From randomly selected $64 \mathrm{~F}_{2}$ plants, we obtained $24 \mathrm{WT}$ plants (SRS5/SRS5), 36 heterozygote plants (Srs5/SRS5), and four mutant homozygote plants (Srs5/Srs5) (Figure 3C) with detecting the genotypes by using the PCR markers chr11-7240 and chr11-9030. The average seed lengths of SRS5/SRS5, Srs5/SRS5, and Srs5/Srs5 in the $\mathrm{F}_{2}$ population were $7.40 \pm 0.15 \mathrm{~mm}, 6.67 \pm 0.24 \mathrm{~mm}$, and $5.84 \pm$ $0.13 \mathrm{~mm}$, respectively (Figure $3 \mathrm{C}$ ). Since Srs $5 / S R S 5$ plants show seed lengths in-between those of SRS5/SRS5 and Srs5/Srs 5 plants, the Srs 5 mutant gene is considered to act as a semi-dominant manner (Figures $1 \mathrm{~A}$ and $1 \mathrm{D}$ and $3 \mathrm{C}$ ). The molecular markers chr11-7240 and chr119030 were used as selection markers for large-scale

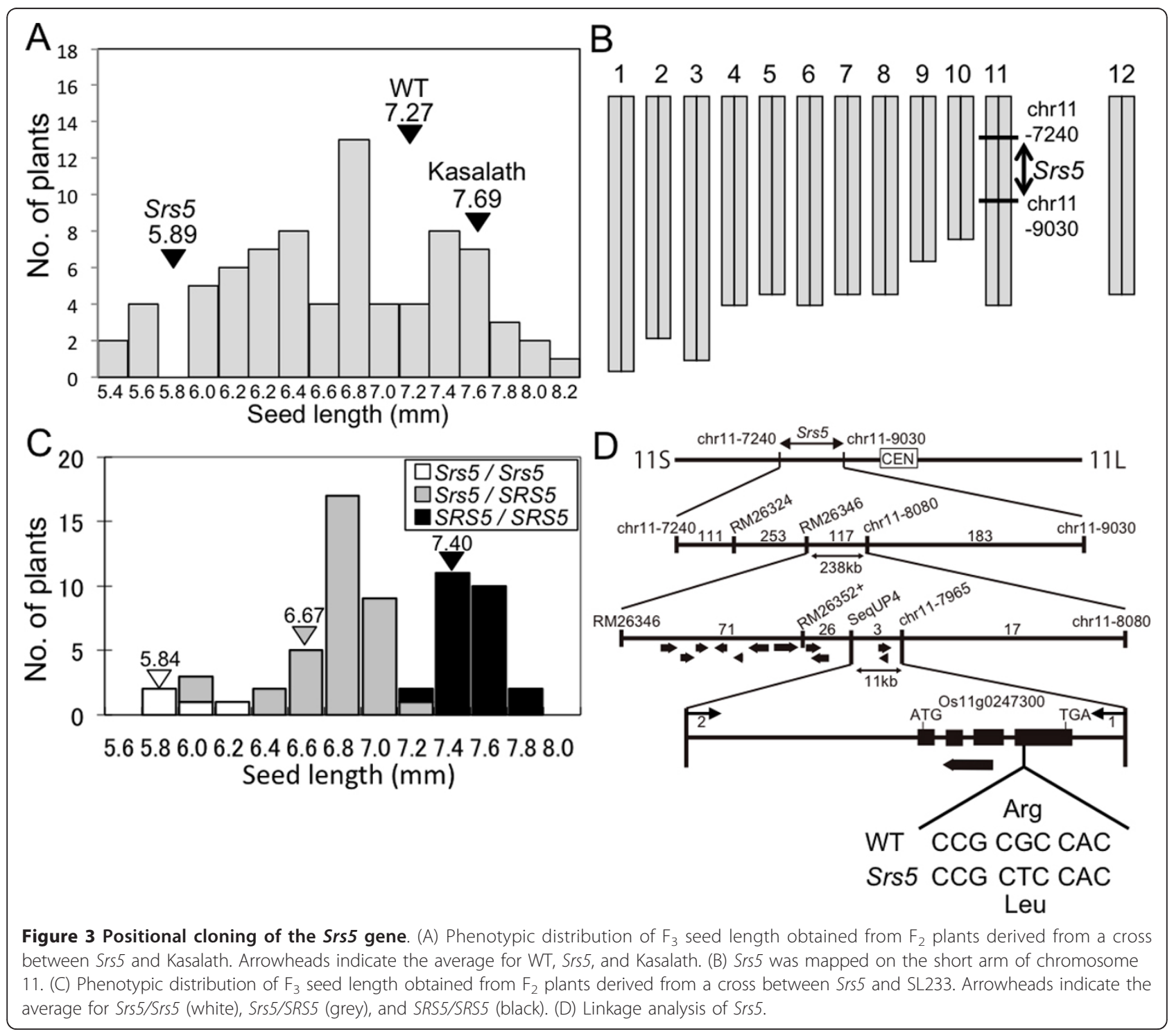


mapping (Figure 3B and 3D). We selected 664 from 5184 $\mathrm{F}_{2}$ recombinant plants that possessed chromosomal recombination within these two markers. We designed new molecular markers between chr11-7240 and chr11-9030, and 664 plants were used for high-resolution mapping (Figure 3D). The genotypes of $F_{2}$ plant were confirmed by observation of the seed of $F_{3}$ and $F_{4}$ progeny. Finally, we obtained three recombinants within the $11 \mathrm{~kb}$ region including two predicted genes (RAPDB http://rapdb.dna.affrc.go.jp/) (Figure 3D). Sequence analysis revealed only one single nucleotide polymorphism (SNP) in Os11g0247300 (Figure 3D). This mutation was resulted in an amino-acid substitution from Arg to Leu in residue 308 (Figure 3D).

\section{Complementation test}

To confirm the SNP as causal mutation of Srs5, we performed a complementation test. The WT SRS5 gene was transformed into the Srs 5 mutant. The seeds of positive transgenic plants were significantly longer than the seeds of those containing empty vector (Figure 4A and 4C). The dwarf phenotype was also rescued (Figure 4B), but phenotype of transgenic plant was not completely same as wild type.
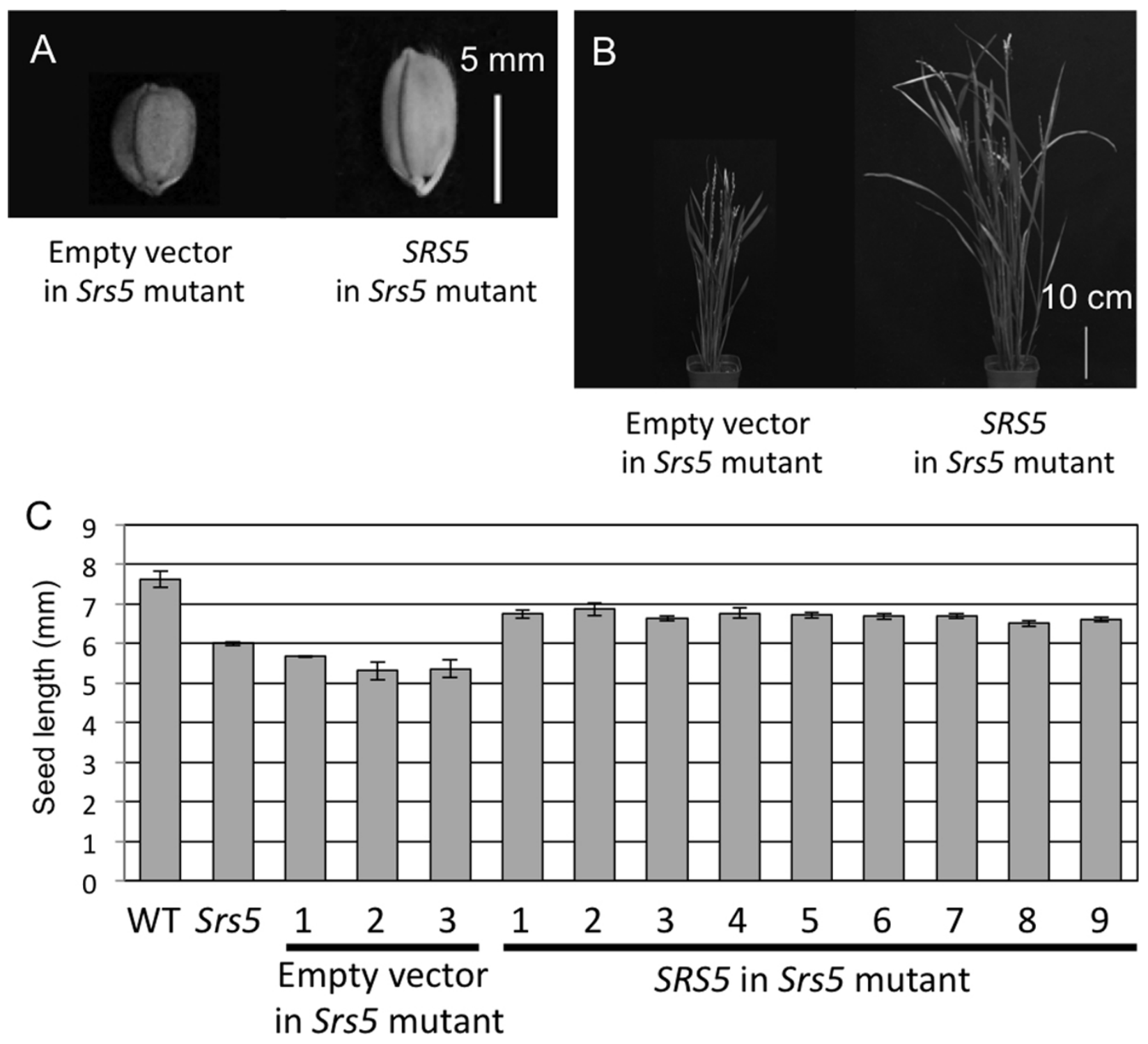

Figure 4 Srs5 complementation test. (A) Seed morphology of transgenic plants. Bar $=5 \mathrm{~mm}$. (B) Gross morphology of transgenic plants. Bar $=$ $10 \mathrm{~cm}$. (C) Seed length of transgenic plants. Numbers on graphs in (C) indicate averages \pm S.D. 


\section{SRS5 expression}

Accumulation of SRS5 mRNAs during the rice life cycle was investigated. SRS5 mRNA accumulated in the shoot apex, young panicles, and spikelets in both the WT and the Srs5 mutant (Figure 5A). In spikelets, accumulation of SRS5 mRNA was higher in the Srs5 mutant than in the WT (Figure 5A). Additionally, we compared the expression levels of SRS5 and BRI1 genes among Srs5, $d 1-1$, and d61-2 mutants. Interestingly, we detected higher accumulation of SRS5 and
BRI1 genes in both of Srs5 and d61-2 mutants (Figure $5 \mathrm{~B}$ and $5 \mathrm{C})$. In $d 1-1$ mutant, the mRNA amounts of these genes were same level as WT (Figure 5B and $5 \mathrm{C}$ ).

\section{Srs5 regulates cell elongation independently of BR signal}

\section{transduction}

Since Srs5 exhibits shorter cells, as does $d 61-2$, we produced a double mutant by crossing to determine whether these two genes have epistasis. The double mutant showed shorter seed length (Figure 6A and 6C)

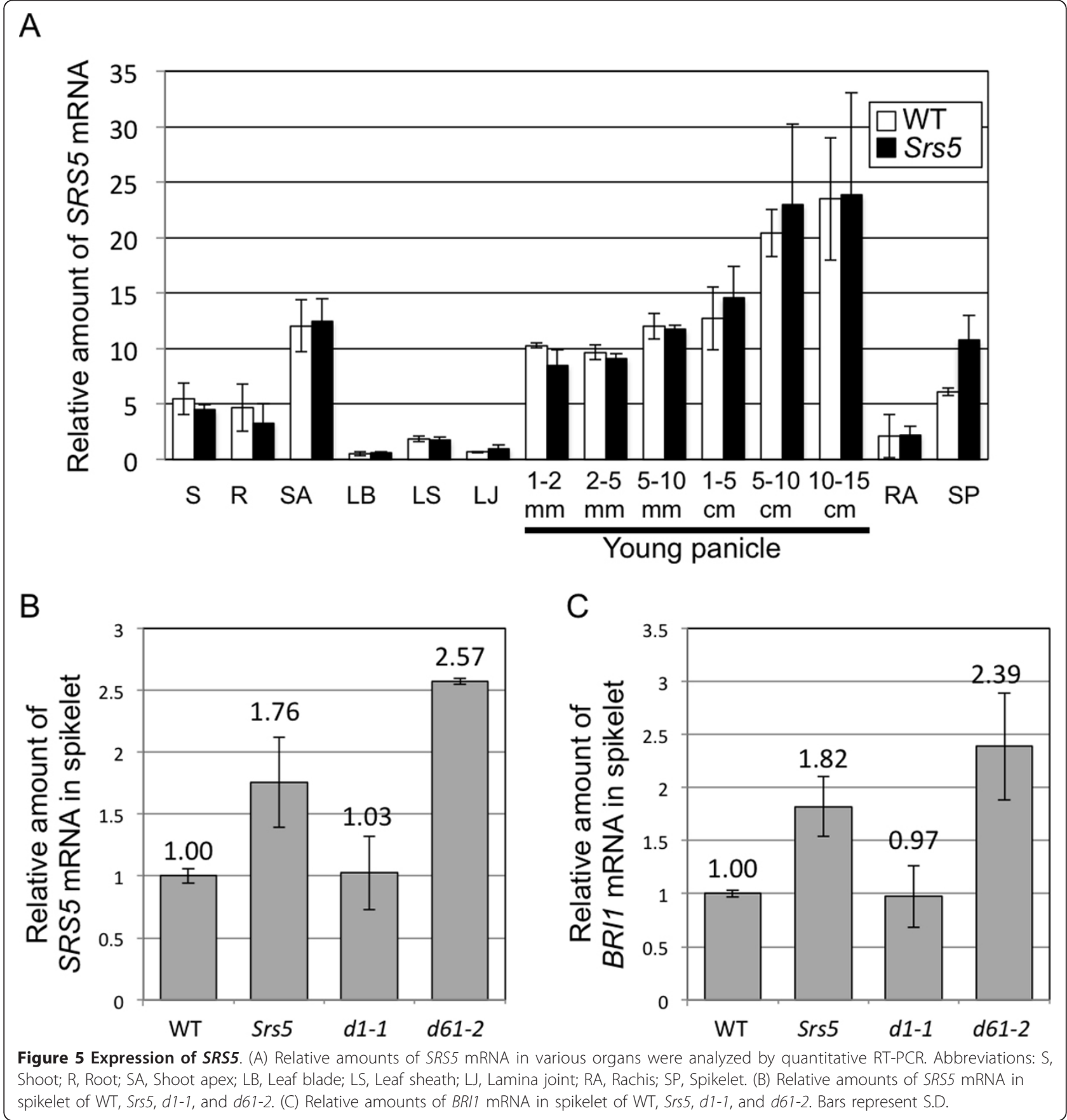



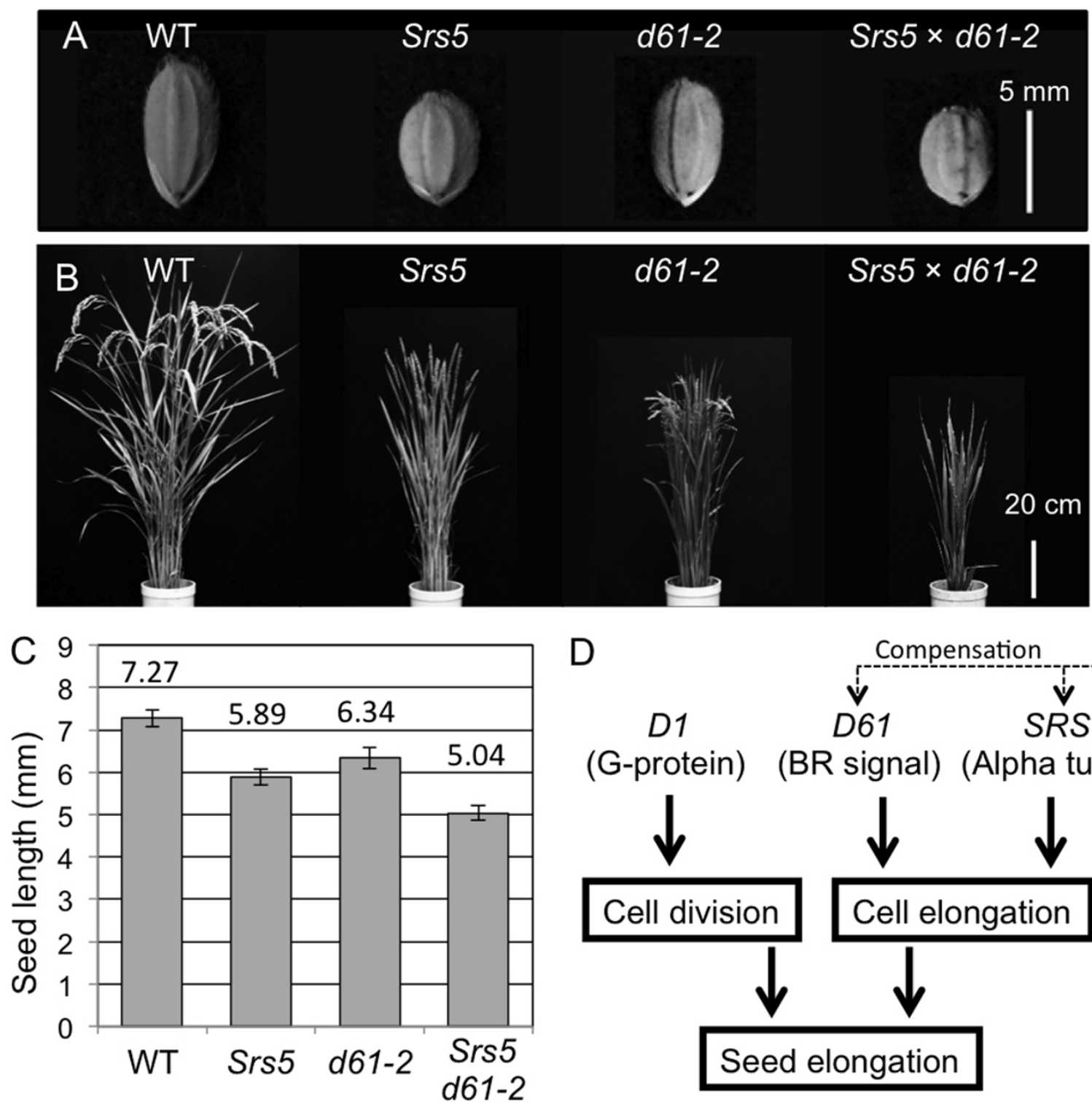

D

D1 (G-protein)

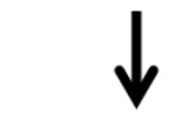

Cell division

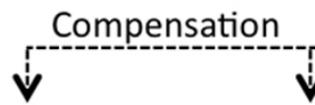

SRS5

(BR signal) (Alpha tubulin)

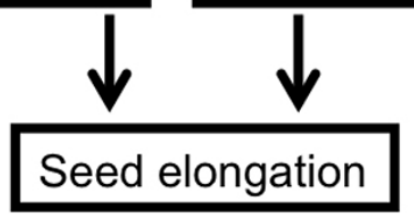

Figure 6 Epistatic test of Srs5 and d61-2. (A) Seed morphology of T65, Srs5, d61-2, and Srs5 xd61-2. Bar = 5 mm. (B) Gross morphology of T65, Srs5, d61-2, and Srs5 xd61-2. Bar = $20 \mathrm{~cm}$. (C) Seed length of T65, Srs5, d61-2, and Srs5×d61-2. Numbers indicate averages \pm S.D. (D) Genetic model of SRS5 function during seed elongation in rice.

and plant height than both parent mutants (Figure 6B). This result indicates that SRS5 and D61 regulate cell elongation independently during seed formation.

\section{Discussion}

In linkage analysis, we could not clearly distinguish seed size in $\mathrm{F}_{2}$ seed derived from distant cross between Srs5 and Kasalath. This was likely to be caused by background difference between indica and japonica. To overcome this, we used a chromosome segment substitution line (CSSL), which is a plant series that possesses relatively large chromosome segments of donor parent chromosomes in the recurrent parental chromosome background (Yano and Sasaki 1997, Yano 2001, Ebitani et al. 2005, Ashikari and Matsuoka 2006, Fukuoka et al. 2010). CSSLs can be used to achieve high accuracy in phenotyping in $F_{2}$ populations. In fact, we could make classification of two seed size, wild and mutant type, in the $F_{2}$ population derived from a cross between Srs 5 and a CSSL.

Genetic analysis of the $\mathrm{F}_{2}$ population derived from a cross between Srs5 and SL233 demonstrated that the Srs5 gene act as semi-dominant manner. This semidominant effect was also confirmed in complementation 
test. Although The Srs5 mutants carrying WT SRS5 gene showed longer seeds than that of the plants containing empty vector, the degree of recovery was not completely same as WT (Figure 4B). The reason that the rescue by WT SRS5 gene was partial may be due to compete between WT and mutation gene products or incomplete conformation of the tubulin complex.

In this study, we demonstrated that the SRS5 gene encodes alpha-tubulin, which has been reported to be the causal gene of the rice mutation $\underline{\text { Twisted }} \underline{\mathrm{d}}$ warf 1(Tid1) (Sunohara 2009). The Tid1 mutation acts as a semidominant gene by affecting the interaction of alpha and beta tubulin. Since Srs 5 was also a semi-dominant mutation, it was likely caused by incomplete conformation of the tubulin complex. Tid1 shows right helical growth, in addition to a semi-dominant dwarf phenotype. Additionally, Arabidopsis Lefty 1 and Lefty 2 mutations in genes orthologous to SRS5 also show semi-dominant and left helical growth (Thitamadee et al. 2002). These two mutants were gain-of-function alleles and exhibited similar twisted plant phenotypes. As the Srs 5 mutant does not exhibit a twisted phenotype, different mutations in alpha-tubulin seem to lead to different phenotypes. In spikelets, higher accumulation of SRS5 mRNA was detected in the Srs5 mutant than in the WT (Figure 5A). This seems to compensate for the reduced function of alpha-tubulin protein. Higher expression of SRS5 was also detected in $d 61-2$ but not in $d 1-1$ (Figure 5B and $5 C)$. Furthermore, BRI1 gene highly expresses in Srs 5 and $d 61-2$ but not in $d 1-1$ (Figure $5 B$ and $5 C$ ). These results suggest that the expression of SRS5 and BRI1 genes are compensated by sensing the cell elongation inhibition in the SRS5 and d61-2 mutants, although SRS5 and BRI1 genes regulate cell elongation independently (Figure 6D). Three other alpha-tubulin genes are present in the rice genome, and they share a high homology (Sunohara et al. 2009). In organs that exhibited no significant change in phenotype in the Srs5 mutant, these alpha-tubulins might work redundantly to maintain rice body planning.

\section{Conclusions}

Our study demonstrated that short seed mutants can be classified into two types: those with reduced cell numbers, e.g., $d 1$, and those with reduced cell length, e.g., $d 61$ (Figure 2A, C, and 2D). This facilitates classification of novel seed mutants. The short seed phenotype of Srs 5 was demonstrated to be caused by reduced cell length, as in $d 61$; however, the additive phenotype of the double mutant indicated that SRS5 and D61 regulate seed length via different mechanisms. To evaluate the mechanisms regulating seed length, observation of microtubule arrangement and analysis of double mutants among srs1, srs3, Srs5, and various BR mutants need to be performed.

\section{Methods}

\section{Plant materials and growth conditions}

Kyudai No. 37 was first identified at Kyushu University and maintained in Togo Field, Nagoya University. Its genetic background is unknown. A japonica cultivar, Taichung 65, was used as the WT plant. $d 1-1$ was identified as spontaneous mutant 'Daikoku' and substituted its genetic background into Taichung 65 by backcrossing with Taichung 65 as a recurrent parent at Kyushu University. d61-2 was obtained by MNU treatment of Taichung $65 . \mathrm{F}_{2}$ and two parental lines were sown at the beginning of April. The seedlings of all plants were transplanted at the beginning of May into a paddy field at the Research Center for Bioresources Development in Fukui, Japan. They were then grown under natural conditions. Transgenic plants were grown in a closed greenhouse under natural sunlight. Room temperature was maintained at $30^{\circ} \mathrm{C}$ from $09: 00$ to $18: 00$ and $25^{\circ} \mathrm{C}$ from 18:00 to 09:00.

\section{Linkage analysis of SRS5}

For mapping, $F_{2}$ plants derived from a cross between Srs5 (japonica) and Kasalath (indica) were used. Genomic DNA was extracted from fresh leaf tissues of $13 F_{2}$ plants that exhibited the small and round seed phenotype by the CTAB method. The genetic linkage between the Srs5 locus and molecular markers was determined using the sequence tagged site (STS) and cleaved amplified polymorphic sequence (CAPS) markers reported by the Rice Genome Program and microsatellite markers (McCouch et al. 2002). $\mathrm{F}_{2}$ plants derived from a cross between Srs 5 and SL233 were used for fine mapping of Srs5 gene. Recombinant plants possessing a recombination between PCR markers chr11-7240 and chr11-9030 were screened from $5184 \mathrm{~F}_{2}$ plants. Other markers on chromosome 11 were designed by comparing the sequences of Srs 5 and SL233. Information on the PCR markers used in this study is shown in Table 1. Phenotypes were determined using $F_{3}$ seeds obtained from $F_{2}$ plants and $F_{4}$ seeds obtained from $F_{3}$ plants.

\section{Production of transgenic plants}

The BAC clone containing the SRS5 gene was screened from the BAC library (constructed by the CUGI BAC/ EST Resource Center) using four PCR primers, alphatub-5kb-up, alpha-tub-intron, alpha-tub-exon, and alpha-tub-5kb-down.

The BAC clone OSJNBa0014D2 was partially digested by Sau3AI and cloned into the BamHI site of binary vector pYLTAC7 (Liu et al. 1999) (provided by RIKEN BioResource Center). This clone contains the $7.26 \mathrm{~kb}$ upstream region from the transcriptional start site of the $\operatorname{Srs} 5$ gene and the $1.13 \mathrm{~kb}$ downstream region from the end of the 3'UTR region of the SRS5 gene. The 
Table 1 Primer sequences used in this study

\begin{tabular}{|c|c|c|}
\hline & Forward $\left(5^{\prime} \rightarrow 3^{\prime}\right)$ & Reverse $\left(5^{\prime} \rightarrow 3^{\prime}\right)$ \\
\hline chr11-7240 & GTTCATGTCCCTATCGATTC & GAGGACCTTATTGTTTGCC \\
\hline RM26324 & GAGATGGAGGGAGAAGCTACG & GTTCATTGGCATCATCAACC \\
\hline RM26346 & GCGCTTGTAGGAAGTTTAATGG & GTATCAGTGCTGGCTTGTAATACC \\
\hline RM26352+ & GCCTACCTTCAGCTTAAAACA & GTAAGATAAGTAAGACAACGAG \\
\hline SeqUP4 & GTTGTCTITTCCAATTGTAGATA & CATACATAATATCCATAGACTATT \\
\hline chr11-7965 & GACGTTAACTAAGGCTGTGTT & GTTAAGCTGTGTCTAGATCC \\
\hline chr11-8080 & CTCAGTTACTCTGATCTTCC & TCAAGCTTCTGTTCACAAGC \\
\hline chr11-9030 & TGCTCAGACCTTACAATGAG & TCAAACATGCACCAGAGTTC \\
\hline alpha-tub-5kb-up & GTGCTCAAGATGGTCGATGA & GTGCTCAAGATGGTCGATGA \\
\hline alpha-tub-intron & GCCATGATCCGTGCTGAAAT & GCCATGATCCGTGCTGAAAT \\
\hline alpha-tub-exon & CGACAATGAGGCCATCTATG & CGACAATGAGGCCATCTATG \\
\hline alpha-tub-5kb-down & GCTCTCTTCCAGAAATCAAGA & GCTCTCTTCCAGAAATCAAGA \\
\hline RT-alpha-tub & ATGAGGGAGTGCATCTCGAT & CAAGATCGACGAAGACAGCA \\
\hline RT-OsUbiquitin & CTTGGTCGTGTCCCGTTTC & TTCTTCCATGCTGCTCTACCAC \\
\hline
\end{tabular}

binary vector was transformed into Agrobacterium tumefaciens strain EHA105 (Hood 1993) by electroporation, and Srs 5 mutants were transformed as reported previously (Ashikari et al. 2005). Srs5 mutants containing empty vectors were used as controls.

\section{RNA isolation and RT-PCR}

Total RNA was extracted using the RNeasy Mini Kit (QIAGEN, Hilden, Germany). cDNAs were synthesized from total RNA using the SuperScript III system (Invitrogen, Carlsbad, CA, USA).

For quantification of SRS5 mRNA, real-time RT-PCR was carried out using SYBR Premix Ex Taq TM II (TAKARA Bio, Inc., Tokyo, Japan). Two primers, RTalpha-tub, were used to quantify Srs 5 expression; a further two, RT-OsUbiquitin, were used to quantify OsUbiquitin1 expression (accession No. Os06g0681400). The Thermal Cycler Dice Real Time System (TAKARA Bio, Inc.) was used for quantification for real-time RTPCR.

\section{Acknowledgements}

This work was supported in part by the Funding Program for Next Generation World-Leading Researchers (NEXT Program) [GS-024] and the Ministry of Agriculture, Forestry and Fisheries of Japan [Genomics for Agricultural Innovation IPG-0002].

\section{Author details}

${ }^{1}$ Faculty of Biotechnology, Fukui Prefectural University, 4-1-1 Kenjojima, Matsuoka, Eiheiji-Town, Fukui 910-1195, Japan ${ }^{2}$ Institute of the Society for Techno-innovation of Agriculture, Forestry and Fisheries, 446-1 Ippaizuka, Kamiyokoba, Tsukuba, Ibaraki 305-0854, Japan ${ }^{3}$ Laboratory of Synaptic Plasticity and Connectivity RIKEN Brain Science Institute, 2-1 Hirosawa, Wako, Saitama 351-0198, Japan ${ }^{4}$ National Institute of Agrobiological Science, 2-1-1 Kannondai, Tsukuba, Ibaraki 305-8602, Japan ${ }^{5}$ National Institute of Agrobiological Science, 1-2 Ohwashi, Tsukuba, Ibaraki 305-8634 Japan ${ }^{6}$ Bioscience and Biotechnology Center, Nagoya University, Furo, Chikusa, Nagoya, Aichi 464-8601, Japan

\section{Authors' contributions}

SS carried out molecular genetic studies, expression analysis, electron microscopic analysis, and transgenic analysis, and wrote manuscript. IK carried out molecular genetic studies. TA carried out molecular genetic studies. MY carried out molecular genetic studies and wrote manuscript. HK provided all plant materials KM designed research, carried out molecular genetic studies, expression analysis, electron microscopic analysis, and transgenic analysis, and wrote manuscript. YI designed research and wrote manuscript. All authors read and approved the final manuscript.

\section{Competing interests}

The authors declare that they have no competing interests.

Received: 12 December 2011 Published: 27 February 2012

\section{References}

Abe Y, Mieda K, Ando T, Kono I, Yano M, Kitano H, Iwasaki Y (2010) The SMALL AND ROUND SEED1 (SRS1/DEP2) gene is involved in the regulation of seed size in rice. Genes Genet Syst 85:327-39

Ashikari M, Sakakibara H, Lin S, Yamamoto T, Takashi T, Nishimura A, Angeles ER, Qian Q, Kitano H, Matsuoka M (2005) Cytokinin oxidase regulates rice grain production. Science 309:741-5

Ashikari M, Matsuoka M (2006) Identification, isolation and pyramiding of quantitative trait loci for rice breeding. Trends Plant Sci 11:344-50

Ashikari M, Wu J, Yano M, Sasaki T, Yoshimura A (1999) Rice gibberellininsensitive dwarf mutant gene Dwarf 1 encodes the alpha-subunit of GTPbinding protein. Proc Natl Acad Sci USA 96:10284-9

Ebitani T, Takeuchi Y, Nonoue Y, Yamamoto T, Takeuchi K, Yano M (2005) Construction and evaluation of chromosome segment substitution lines carrying overlapping chromosome segments of indica rice cultivar Kasalath in a genetic background of japonica elite cultivar Koshihikari. Breed Sci 55:65-73

Fukuoka S, Nonoue Y, Yano M (2010) Germplasm enhancement by developing advanced plant materials from diverse rice accessions. Breed Sci 60:509-517

Fujisawa Y, Kato T, Ohki S, Ishikawa A, Kitano H, Sasaki T, Asahi T, Iwasaki Y (1999) Suppression of the heterotrimeric $G$ protein causes abnormal morphology, including dwarfism, in rice. Proc Natl Acad Sci USA 96:7575-80

Hong Z, Ueguchi-Tanaka M, Fujioka S, Takatsuto S, Yoshida S, Hasegawa Y, Ashikari M, Kitano H, Matsuoka M (2005) The rice brassinosteroid-deficient dwarf2 mutant, defective in the rice homolog of Arabidopsis DIMINUTO/ DWARF1, is rescued by the endogenously accumulated alternative bioactive brassinosteroid, dolichosterone. Plant Cell 17:2243-54

Hong Z, Ueguchi-Tanaka M, Umemura K, Uozu S, Fujioka S, Takatsuto S, Yoshida S, Ashikari M, Kitano H, Matsuoka M (2003) A rice brassinosteroiddeficient mutant, ebisu dwarf (d2), is caused by a loss of function of a new member of cytochrome P450. Plant Cell 15:2900-10 
Hood EE, Gelvin SB, Melches LS, Hoekema A (1993) New Agrobacterium helper plasmids for gene transfer to plants. Transgenic Res 2:208-218

Izawa Y, Minami M, Ohki S, Iwasaki Y (2010) Expression profile of the a subunit of the heterotrimeric $\mathrm{G}$ protein in rice. Plant Signal Behav 5:845-7

Kitagawa K, Kurinami S, Oki K, Abe Y, Ando T, Kono I, Yano M, Kitano H, Iwasaki Y (2010) A novel kinesin 13 protein regulating rice seed length. Plant Cell Physiol 51:1315-29

Liu YG, Shirano Y, Fukaki H, Yanai Y, Tasaka M, Tabata S, Shibata D (1999) Complementation of plant mutants with large genomic DNA fragments by a transformation-competent artificial chromosome vector accelerates positional cloning. Proc Natl Acad Sci USA 96:6535-40

McCouch SR, Teytelman L, XU Y, Lobos KB, Clare K, Walton M, Fu B, Maghirang R, Li Z, Xing Y, Zhang Q, Kono I, Yano M, Fjellstrom R, DeClerck G, Schneider D, Cartinhour S, Ware D, Stein L (2002) Development and mapping of 2240 new SSR markers for rice (Oryza sativa L.). DNA Res 9:199-207

Sunohara H, Kawai T, Shimizu-Sato S, Sato Y, Sato K, Kitano H (2009) A dominant mutation of TWISTED DWARF 1 encoding an alpha-tubulin protein causes severe dwarfism and right helical growth in rice. Genes Genet. Syst 84:209-18

Tanabe S, Ashikari M, Fujioka S, Takatsuto S, Yoshida S, Yano M, Yoshimura A, Kitano H, Matsuoka M, Fujisawa Y, Kato H, Iwasaki Y (2005) A novel cytochrome P450 is implicated in brassinosteroid biosynthesis via the characterization of a rice dwarf mutant, dwarf11, with reduced seed length. Plant Cell 17:776-90

Thitamadee S, Tuchihara K, Hashimoto T (2002) Microtubule basis for left-handed helical growth in Arabidopsis. Nature 417:193-6

Yamamuro C, Ihara Y, Wu X, Noguchi T, Fujioka S, Takatsuto S, Ashikari M, Kitano H, Matsuoka M (2000) Loss of function of a rice brassinosteroid insensitive1 homolog prevents internode elongation and bending of the lamina joint. Plant Cell 12:1591-606

Yano M (2001) Genetic and molecular dissection of naturally occurring variation. Curr Opin Plant Biol 4:130-5

Yano M, Sasaki T (1997) Genetic and molecular dissection of quantitative traits in rice. Plant Mol Biol 35:145-53

doi:10.1186/1939-8433-5-4

Cite this article as: Segami et al:: Small and round seed 5 gene encodes alpha-tubulin regulating seed cell elongation in rice. Rice 2012 5:4.

\section{Submit your manuscript to a SpringerOpen ${ }^{\circ}$ journal and benefit from:}

- Convenient online submission

- Rigorous peer review

- Immediate publication on acceptance

- Open access: articles freely available online

- High visibility within the field

- Retaining the copyright to your article

Submit your next manuscript at $\gg$ springeropen.com 